\title{
Long-term prognosis of breast cancer: an analysis of 462 patients in a general hospital in south east Netherlands
}

\author{
Henk W. Nab, ${ }^{*}$ Huub M. Kluck, ${ }^{*} \ddagger$ Emiel J. T. Rutgers, $\S$ \\ Jan-Willem W. Coebergh* $\dagger$ and Wim C. J. Hop $\dagger$ \\ ${ }^{*}$ Comprehensive Cancer Centre South, Eindhoven, $\dagger$ Department of Epidemiology \& Biostatistics, \\ Erasmus University Medical School, Rotterdam, $\ddagger$ Sint Joseph Hospital, Veldhoven, and \\ §The Netherlands Cancer Institute (Antoni van Leeuwenhoek Huis). Amsterdam.
}

The Netherlands

\begin{abstract}
In this study the long-term prognosis was analysed of all $\mathbf{4 6 2}$ consecutive female breast cancer patients who were diagnosed and carefully staged between 1970 and 1980 in a 600-bed community hospital in Eindhoven, south east Netherlands. Follow-up of recurrence and causes of death was obtained until 1 January 1993 . Observed survival rates at 5, 10 and 20 years were $66 \%, 45 \%$ and $32 \%$, respectively, and the corresponding breast cancer-specific survival rates were $71 \%, 54 \%$ and $44 \%$. The yearly risk for a recurrence of breast cancer after treatment steadily decreased from $10 \%$ the first year to $1 \%$ after 10 years.

In a multivariate survival analysis both tumour size and nodal status appeared to be equally important prognostic factors in the first 5 years after diagnosis. After 5 years only tumour size had independent prognostic value, which was not significant any more after 10 years. In patients with a tumour size $\leqslant 2 \mathrm{~cm}$ and without lymph node involvement at diagnosis, the risk for a recurrence was found to be negligible after 10 years. Those patients may be considered cured, although a search for early diagnosis of a second primary breast cancer in this group is still advisable.
\end{abstract}

Key words: breast cancer; prognostic factors; survival.

\section{Introduction}

Although the percentage of long-term survivors after breast cancer is relatively high, a cure is unlikely to be confirmed before at least 15 years follow-up. ${ }^{1-6}$ Because decisions on continuation of routine control visits should be well founded, more detailed knowledge regarding the time periods during which prognostic factors have their greatest influence may be of practical value. ${ }^{7}$ This may also add to the knowledge on the related biological mechanisms.

However, in breast cancer, contrary to factors which predict short-term survival, little is known about the factors predicting long-term survival. 6,8-10

This report presents survival rates of carefully staged and documented breast cancer patients diagnosed between 1970 and 1980 in a general hospital in south east Netherlands, with follow-up until 1993. The prognostic potential of tumour size, nodal status and age group is investigated within different follow-up intervals.

\section{Material and methods}

The study includes all patients with breast cancer diagnosed between 1970 and 1980 in the Sint Joseph Hospital in

Correspondence: H. W. Nab, MD, Comprehensive Cancer Centre South, P.O. Box 231, 5600 AE Eindhoven, The Netherlands.
Eindhoven (now Veldhoven), a community hospital of about 600 beds. Clinical staging was done according to the UICC classification, 1968." Tumour size was measured by the pathologist and divided into three categories: pT $1(\leqslant 2 \mathrm{~cm})$, pT2 $(2-5 \mathrm{~cm})$, or pT3 $(>5 \mathrm{~cm})$. Axillary lymph node status was divided into three categories: pNO (lymph node negative), pN1 (lymph node positive, without involvement of the apex and without extra nodal growth), or pN2 (lymph node positive with involvement of the apex of the axilla, or with extra nodal growth). Up to 1974 pre-operative biopsy of the apex lymph nodes was usual, followed by a complete axillary clearance when lymph nodes were negative on frozen section. After 1974 an immediate complete axillary dissection en bloc with a mastectomy was common practice. The presence of distant metastasis was screened by clinical and laboratory investigations, routine chest radiographs, and by more advanced techniques, if indicated. Overall, four surgeons were involved in the treatment of breast cancer patients, who mainly uised radical (before mid-1976) and modified radical (after mid-1976) mastectomy. Patients with central or medial tumour localization received adjuvant radiotherapy to the parasternal lymph nodes. Patients with pT3 tumours and/or three or more axillary lymph nodes received radiotherapy to the supraclavicular, axillary, parasternal lymph nodes and the chest wall. In 1979 adjuvant CMF treatment was introduced for premenopausal axillary lymph node positive patients, and only 18 patients received this 
therapy. After primary treatment patients were seen in the out-patient clinic every 3 months in the first 2 years, every 6 months up to 5 years, and annually thereafter. ${ }^{2}$ In this period of diagnosis in Eindhoven there were two other general hospitals. To our knowledge there was no particular case selection or referral to other institutions for a substantial number of patients.

Active follow-up was carried out up to 1 January 1993. Causes of death could be traced. Death from breast cancer included only those patients with known metastases. Only 12 patients $(3 \%)$ were lost to follow-up after variable intervals of observation.

Observed (actuarial) survival curves were computed, ${ }^{13}$ according to age category (under 50, 50-65, 65+ years), tumour size and nodal status. Breast cancer-specific survival was calculated by considering patients withdrawn from the study at the moment of non-breast cancer death. Diseasefree survival was calculated for patients without distant disease at diagnosis to recurrence, the end of the study, or to death. Differences in survival were assessed by the log-rank test, also after adjustment by stratification for other variables. The Cox proportional hazards model was used to simultaneously evaluate the prognostic importance of age, tumour size and nodal status. ${ }^{14}$ This was done separately for the first and second 5-year follow-up interval, and for the subsequent 10-year interval. Other statistical methods are indicated in the text. $P$-values given are two-sided; five percent was considered the limit of significance.

\section{Results}

The number of patients according to clinical stage, pathological tumour size and lymph node status is listed in Table 1. Overall, 462 patients were included with a mean age at diagnosis of 57 years (range, 23 to 90 years). Age category did not correlate with pathological tumour size or lymph node status (Kruskal-Wallis tests, $P$-values $>0.1$ ). During the first 5 years of follow-up, 158 women were reported dead: $132(84 \%)$ due to breast cancer. After 10 years another 99 women had died, $70(71 \%)$ due to breast cancer; and after

Table 1. Characteristics of the patient group

\begin{tabular}{lcc}
\hline & Number of patients & $\%$ \\
\hline Clinical stage & 103 & \\
I & 204 & 22 \\
II & 115 & 44 \\
III & 34 & 25 \\
IV & 6 & 7 \\
Unknown & & 1 \\
Tumour size & 134 & \\
pT1 & 246 & 29 \\
pT2 & 60 & 53 \\
pT3 & 22 & 13 \\
Unknown & & 5 \\
Lymph nodes & 231 & \\
pN0 & 101 & 50 \\
pN1 & 101 & 22 \\
pN2 & 29 & 22 \\
Unknown & 462 & 6 \\
Total & & 100 \\
\hline
\end{tabular}

20 years another 37 women had died, $24(65 \%)$ due to breast cancer.

Observed survival rates for the total group at 5, 10, and 20 years were $66 \%, 45 \%$, and $32 \%$, respectively; the corresponding breast cancer-specific survival rates were $71 \%$, $54 \%$ and $44 \%$. In patients without distant metastasis at diagnosis the risk for a recurrence steadily decreased from an annual $10 \%$ in the first two years after treatment to about $1 \%$ after 10 years; thereafter, this decrease continued. Clinical stage predicted breast cancer survival very well $(P<0.001$, Fig. 1$)$.

Within the node negative patient group (pN0), prognosis in $\mathrm{pT} 1$ patients was significantly better than in $\mathrm{pT} 2$ and pT3 patients $(P<0.01)$, but prognosis was not significantly different between pT2 and pT3 patients $(P>0.2$; Fig. 2$)$. Among the node-positive patients, pTl patients had a more favourable prognosis than pT2 and pT3 patients $(P<0.01)$, and pT2 than pT3 patients $(P<0.01$, Fig. 3$)$. After 10 years of follow-up, $72 \%$ of the pT1N0 patients $(n=79)$, were free of recurrence; of these women, only one patient developed a recurrence afterwards. In fact, of the 79 pTIN0 patients surviving for 10 years, there was only one recurrence in the remaining 488 cumulative follow-up years.

In the first 5 years of follow-up both tumour size and lymph node status were significant prognostic factors for disease-free survival, with approximately equal power in a Cox regression analysis (Table 2). The second 5 years of follow-up, tumour size was again an important prognostic factor, in contrast to nodal status. After 10 years of followup the prognostic effect of tumour size remained, although to a smaller extent. The independent effect of age on prognosis, adjusted for tumour size and nodal status, was very small: only the oldest age group had a better prognosis in the first 5 years of follow-up and a worse prognosis after 10 years of follow-up as compared to the reference category; however, both these estimates were only borderline significant.

\section{Discussion}

The present analysis shows that the prognostic influence of tumour size remains present for a longer time period as compared to nodal status. This finding is in line with the results reported by Toikkanen et al. ${ }^{10}$ Their study among 10-year survivors of breast cancer, showed that tumour size remained a significant prognostic factor after 10 years of follow-up, whereas nodal status only predicted survival for the first 10 years of follow-up. The finding that the prognostic effect of nodal status has disappeared after 5 years is in agreement with the results reported by Lipponen $e t$ al. ${ }^{8}$ They found that the marked prognostic influence of both tumour size and nodal status diminished steadily during the first 5 years of follow-up, becoming non-significant after 5 years of follow-up.

In this analysis of breast cancer survival the influence of prognostic factors considerably changed over time. Therefore, it is advised to distinguish between short follow-up intervals in survival analyses.

An earlier analysis of this patient group showed that the great majority of local recurrences were detected during routine control visits, ${ }^{12}$ and that the intensive search for 


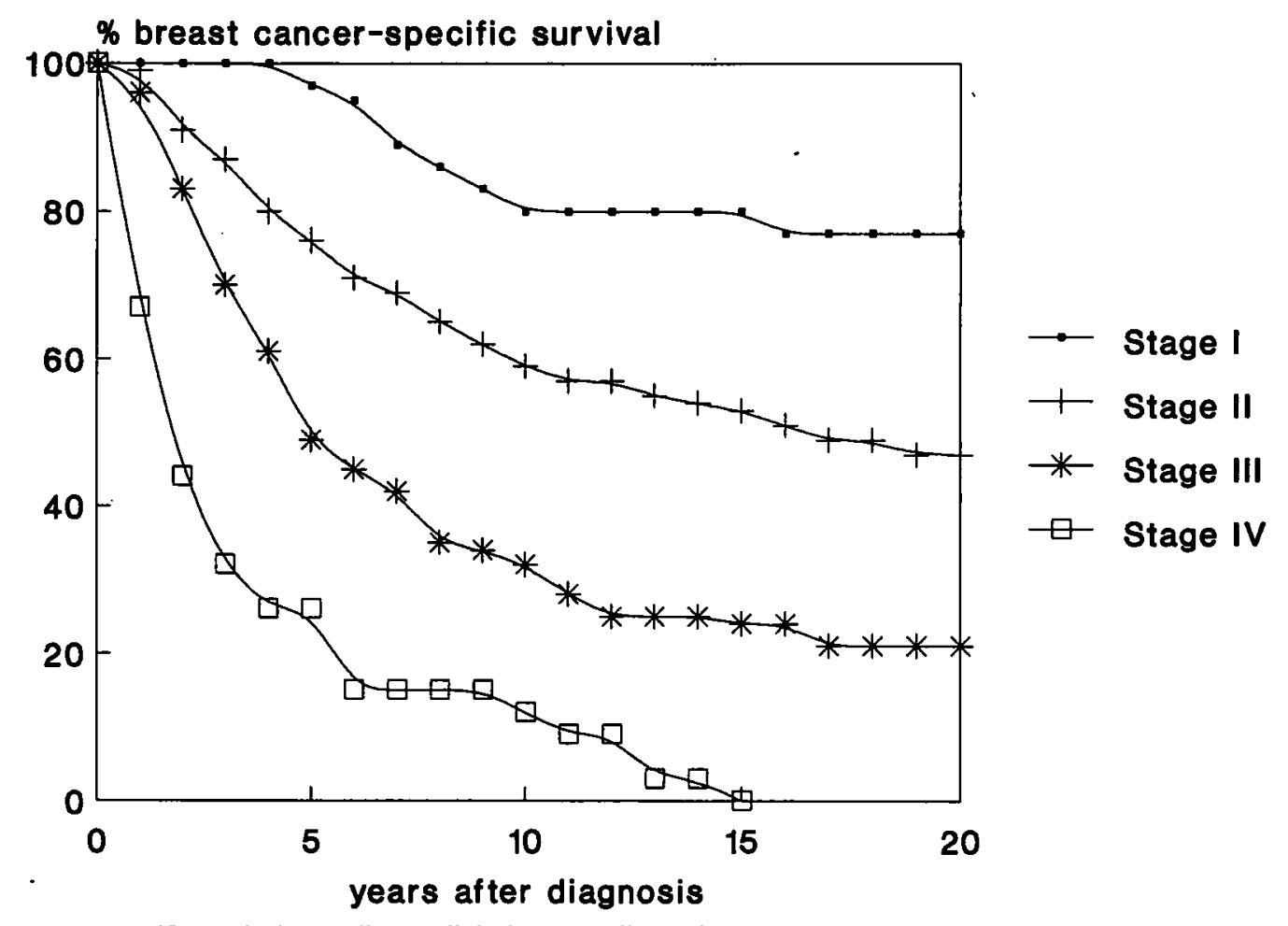

Fig. 1. Breast cancer-specific survival according to clinical stage at diagnosis.

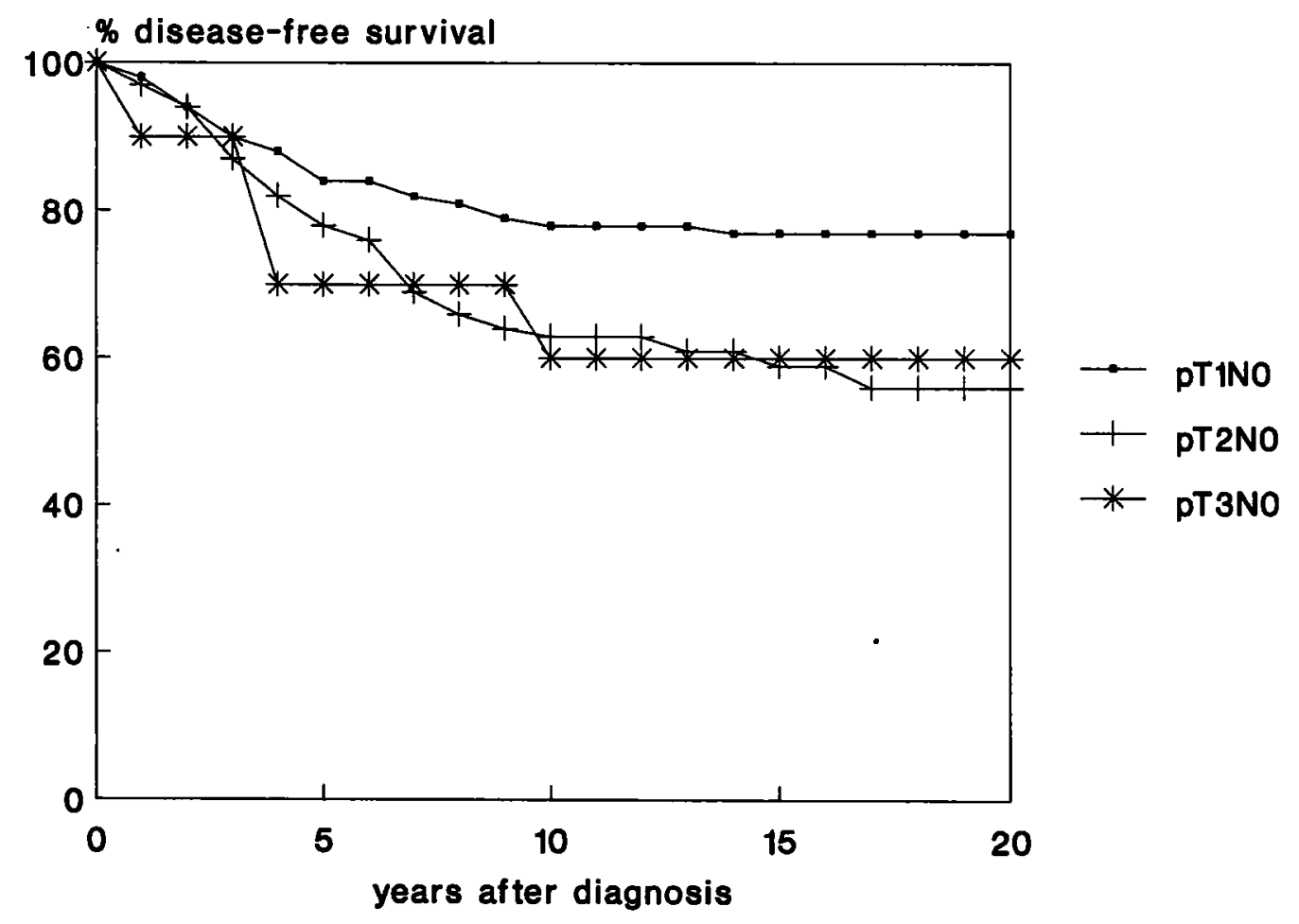

Fig. 2. Disease-free survival of node negative breast cancer according to tumour size. 


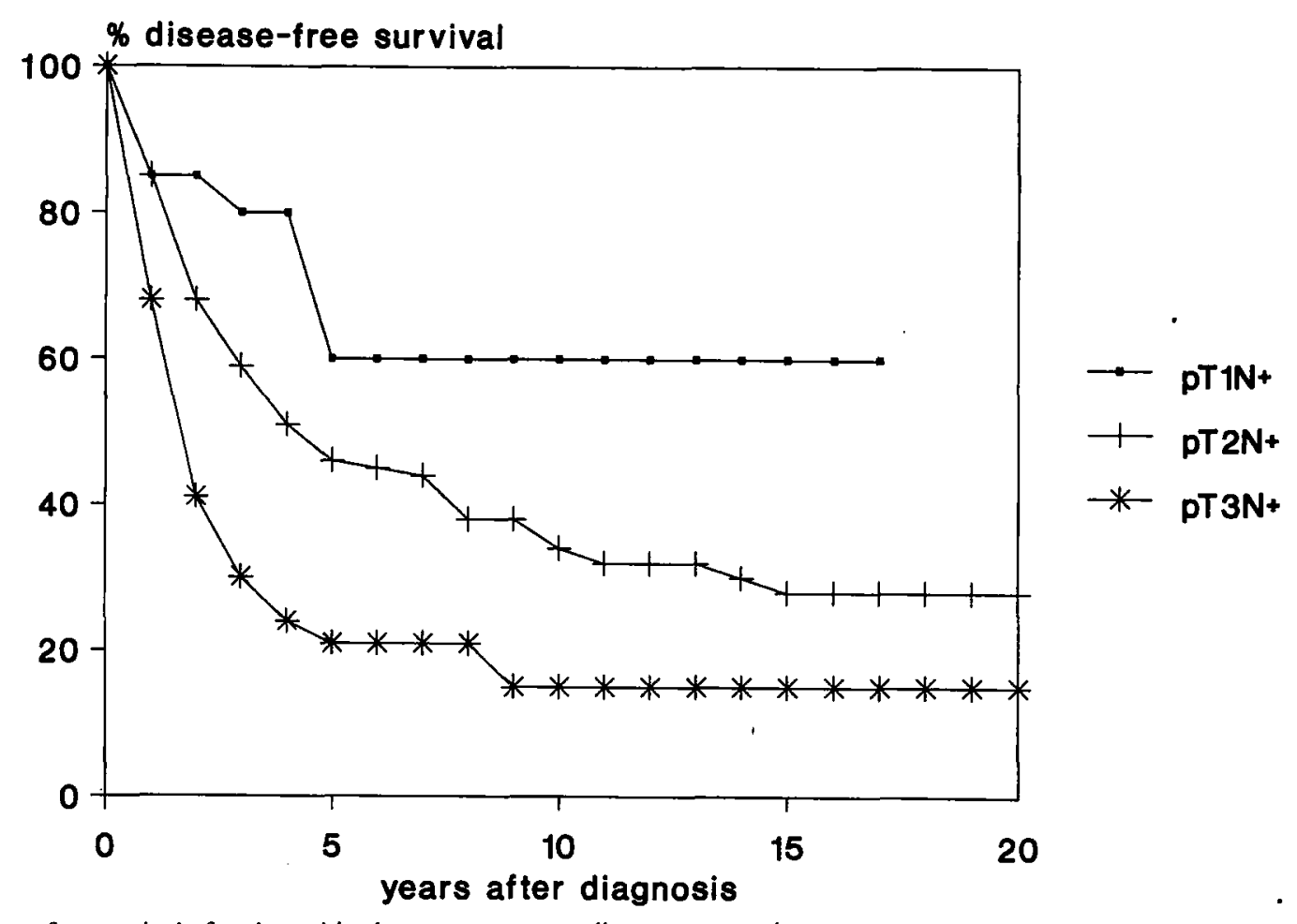

Fig. 3. Disease-free survival of node positive breast cancer according to tumour size.

Table 2. Rate ratios $(95 \% \mathrm{CI})$ for disease recurrence per follow-up interval, according to age, postoperative tumour size and nodal status

\begin{tabular}{llll}
\hline & \multicolumn{3}{c}{ Follow-up interval } \\
\cline { 2 - 4 } Factor & $0-5$ years & $5-10$ years & $10-20$ years \\
\hline $\begin{array}{l}\text { Age group (years) } \\
\quad 50^{*}\end{array}$ & 1 & 1 & 1 \\
$50-65$ & $0.8 \dagger(0.5-1.1)$ & $0.8 \dagger(0.3-1.8)$ & $1.0(0.1-17)$ \\
$65+$ & $0.7 \dagger(0.5-1.0)$ & $1.5 \dagger(0.7-3.5)$ & $10(1.0-104)$ \\
Tumour size & & & 1 \\
pT1* & 1 & 1 & $4.3 \ddagger(0.4-41)$ \\
pT2 & $2.0(1.3-3.1)$ & $6.4 \dagger(2.2-19)$ & \\
pT3 & $3.4(1.9-5.8)$ & $5.7 \S(1.2-26)$ & 1 \\
Nodal status & & & $0.5 \S(0.1-5)$ \\
pN0* & 1 & 1 & \\
pN1 & $1.9(1.3-2.7)$ & $0.9 \dagger(0.4-2.1)$ & \\
pN2 & $3.5(2.4-5.2)$ & $1.2 \dagger(0.4-3.2)$ & \\
\hline
\end{tabular}

* Reference category.

$\dagger$ Estimates are not significantly different from each other.

$\ddagger$ Estimate for $\mathrm{pT} 2$ and $\mathrm{pT} 3$ combined vs $\mathrm{pT} 1$.

$\S$ Estimate for $\mathrm{pN}+\mathrm{vs} \mathrm{pN} 0$.

distant disease by routine follow-up means did not appear to be beneficial to the patients. From this study it was concluded that follow-up after treatment of primary breast cancer should be limited to taking the history, physical examination with emphasis on the loco-regional status, and an annual mammography for the detection of contralateral breast cancer. In the present analysis it appeared that the risk for a recurrence steadily decreased during follow-up, and that after 10 years of follow-up it became very small, particularly in patients with pTIN0 tumours. Weighing the advantage of a very small chance for detecting a recurrence against the disadvantage of many follow-up visits and examinations, we conclude that pT1N0 breast cancer patients who survive for 10 years may be considered cured, with no need for further routine follow-up visits for the detection of metastasis. However, the increased risk of a contralateral breast cancer, ${ }^{15.16}$ may well warrant a search for early diagnosis of a second primary breast cancer. ${ }^{17}$ 


\section{References}

1. Langlands AO, Pocock SJ, Kerr GR, Gore SM. Long-term survival of patients with breast cancer: a study of the curability of the disease. Br Med J 1979; 2: 1247-51.

2. Brinkley D, Haybittle JL. Long-term survival of women with breast cancer. Lancet 1984; i: 1118.

3. Rutgvist LE, Wallgren A. Long-term survival of 458 young breast cancer patients. Cancer 1985; 55: 658-65.

4. Hibberd AD, Horwood LJ, Wells JE. Long term prognosis of women with breast cancer in New Zealand: study of survival to 30 years. Br Med J 1983; 286: 1777-9.

5. Duncan W, Kerr GR. The curability of breast cancer. Br Med $J$ 1976; 2; 781-3.

6. Nab HW, Hop WCJ, Crommelin MA, Kluck HM, van der Heijden LH, Coebergh JWW. Changes in long term prognosis for breast cancer in a Dutch cancer registry. Br Med J 1994; 309: 83-6.

7. Dixon JM, Pager DL, Anderson TJ el al. Long term survivors after breast cancer. Br J Surg 1985; 72: 445-8.

8. Lipponen P, Aaltomaa S, Eskelinen M, Kosma VM. Marin S, Syrjänen $\mathrm{K}$. The changing importance of prognostic factors in breast cancer during long-term follow-up. Int J Cancer 1992; 51: $698-702$

9. Nab HW, Hop WCJ, Crommelin MA, Kluck HM, Coebergh
JWW. Improved prognosis in breast cancer since 1970 in southeastern Netherlands. Br J Cancer 1994; 70: 285-8.

10. Toikkanen SP, Kujari HP, Joensuu H. Factors predicting late mortality from breast cancer. Eur J Cancer 1991; 27: 586-91.

11. Harmer $\mathrm{MH}$, Copeland MM, Costachel O et al. (eds). Breast. TNM Classification of malignant Tumours. International Union Against Cancer. Geneva, 1968; pp. 39-43.

12. Rutgers EJTh, van Slooten EA, Kluck HM. Follow-up after treatment of primary breast cancer. $B r J$ Surg 1989; 76: 187-90.

13. Berkson J, Gage RP. Calculation of survival rates for cancer. Proc Staff Meet Mayo Clin 1950; 25: 270.

14. Cox DR. Regression models and life tables. J R Stat Soc 1972; B34: 187-207.

15. Hislop TG, Elwood JM, Coldman AJ, Spinelli JJ, Worth AJ, Ellison LG. Second primary cancers of the breast: incidence and risk factors. Br J Cancer 1984; 49: 79-85.

16. Horn PL, Thompson WD, Schwartz SM. Factors associated with the risk of second primary breast cancer: an analysis of data from the Connecticut Tumor Registry. J Chron Dis 1987; 40: 1003-11.

17. Mellink WAM, Holland R, Hendrix JHCL, Peeters PHM, Rutgers EJT, van Daal WAJ. A contribution of routine follow-up mammography to an early diagnosis of asynchronous contralateral breast cancer. Cancer 1991; 67: 1844-8.

Accepted for publication 10 October 1994 